\section{ORIGINAL RESEARCH}

\author{
F. Bing \\ R. Doucet \\ F. Lacroix \\ J.P. Bahary \\ T. Darsaut \\ D. Roy \\ F. Guilbert \\ J. Raymond
}

A. Weill

\title{
Liquid Embolization Material Reduces the Delivered Radiation Dose: Clinical Myth or Reality?
}

BACKGROUND AND PURPOSE: To be radiopaque, BAVM embolization products must contain highatomic-number materials, which may also attenuate photon beams delivered with radiosurgery. This "shielding effect" has been invoked to explain why radiation therapy may be less effective for previously embolized BAVMs. To evaluate the impact of embolization material on radiation dose, we measured and compared the dose delivered to the center of an AVM model, before and following embolization with various materials in a LINAC

MATERIALS AND METHODS: Two in vitro AVM models were constructed by drilling interconnected tubular perforations in plastic water phantoms to simulate nidal vessels. Phantoms were designed to allow the positioning of a radiation detector at their center. One model was embolized with Onyx 18 and a second model, with a combination of Indermil, Lipiodol, tungsten powder, and Onyx 18. The radiation delivered was compared between embolized and nonembolized controls following irradiation with a standard 250-cGy dose.

RESULTS: The mean dose of radiation delivered to the model embolized with Onyx alone was $244 \pm$ 5 cGy before and $246 \pm 5$ cGy following embolization. The mean dose of radiation delivered to the model embolized with various agents was $242 \pm 5$ cGy before, and $254 \pm 5$ cGy after embolization.

CONCLUSIONS: Embolic material did not reduce the radiation dose delivered by a LINAC to the center of our experimental BAVM models. The shielding effect may be compensated by scattered and reflected radiation.

ABBREVIATIONS: BAVM = brain arteriovenous malformation; LINAC = linear accelerator; $n$-BCA $=$ $n$-butyl cyanoacrylate; $Z=$ atomic number

$\mathbf{T}$ he main goal of BAVM treatment is to prevent intracranial hemorrhage. The benefit of prophylactic treatment of unruptured BAVMs is uncertain. ${ }^{1}$ Therapeutic management may consist of 1 or a combination of different approaches, including endovascular embolization, surgery, and radiosurgery. The choice of treatment technique depends on the size and location of the BAVM. Embolization alone can occasionally cure BAVMs, but this technique is more frequently used to decrease the risks of surgery or reduce the size before radiation therapy.

BAVM embolization before radiation therapy remains, however, controversial. Some authors claim that embolization before radiation therapy reduces the final obliteration rate. ${ }^{2-6}$ Materials of high atomic number $(Z)$ are added to embolic agents for radiopacity, which may attenuate the dose of radiation delivered in radiosurgical treatments through a "shielding effect," thereby compromising a known determinant of successful BAVM obliteration. ${ }^{7}$

To evaluate the impact of embolization material on the radiation dose delivered, we constructed a BAVM model that permitted the measurement of radiation doses delivered before and following the deposition of embolization material in the simulated BAVM.

Received March 3, 2011; accepted after revision April 25

From the Departments of Radiology (F.B., T.D., D.R., F.G., J.R., A.W.) and Radio-Oncology (R.D., F.L., J.P.B.), Centre Hospitalier Université de Montréal, Montreal, Quebec, Canada.

Please address correspondence to Alain Weill, MD, Department of Radiology, Centre Hospitalier Université de Montréal, 1560 Sherbrooke East, H2L4M1 Montreal, Quebec, CANADA; e-mail: alain.weill.chum@ssss.gouv.qc.ca

http://dx.doi.org/10.3174/ajnr.A2943

\section{Materials and Methods}

Two in vitro BAVM models were constructed by drilling interconnected tubular perforations to simulate nidal vessels in plastic water-calibration phantoms (plastic water, CIRS, Norfolk, Virginia) (Fig 1A). Models were configured to permit positioning of a water-equivalent detector (Gafchromic EBT film; ISP, Wayne, New Jersey) in the center of the phantom.

The first model (A), measuring $2 \times 2 \times 4 \mathrm{~cm}$, with 1 -mm-diameter channels was embolized with Onyx 18 (ev3, Irvine, California), a commonly used liquid embolic agent containing ethylene-vinyl alcohol copolymer, dimethyl-sulfoxide, and tantalum ( $\mathrm{Z}=73$ ) added for radiopacity. Onyx is presented in a micronized powder form (35\% weight/volume). The second model (B), measuring $4 \times 4 \times 8 \mathrm{~cm}$, with 1 - to 5 -mm-diameter channels, was embolized by using various and multiple agents in greater quantity: $n$ BCA (Indermil; Henkel Loctite, Dusseldorf, Germany) with ethiodized oil (Lipiodol, Andre Guerbet, Aulnay-sous-Bois, France) and Onyx 18. We also added tungsten $(Z=74)$ to the embolic material to obtain a more important shielding effect. In clinical practice, Lipiodol ( $Z=53$ for iodine), tantalum, or tungsten is added to $n$-BCA and accounts for the shielding effect. Plain radiographs of the BAVM models were taken to ensure that radiopaque embolization material filled the channels (Fig $1 B$ ).

After appropriate positioning of the water-equivalent detector at the center of the phantom, we irradiated BAVM models with $250 \mathrm{cGy}$, by using a standard 4-arc stereotactic treatment plan on a LINAC (beam energy of 6 $\mathrm{mV}$ ) (Fig $2 A,-B$ ) and compared measured radiation doses for the embolized models and the equivalent nonembolized controls. Each experiment was repeated twice.

\section{Results}

The nonembolized model A controls had a mean dose of radiation delivered of $244 \pm 5$ cGy (247 and 240 cGy for indi- 

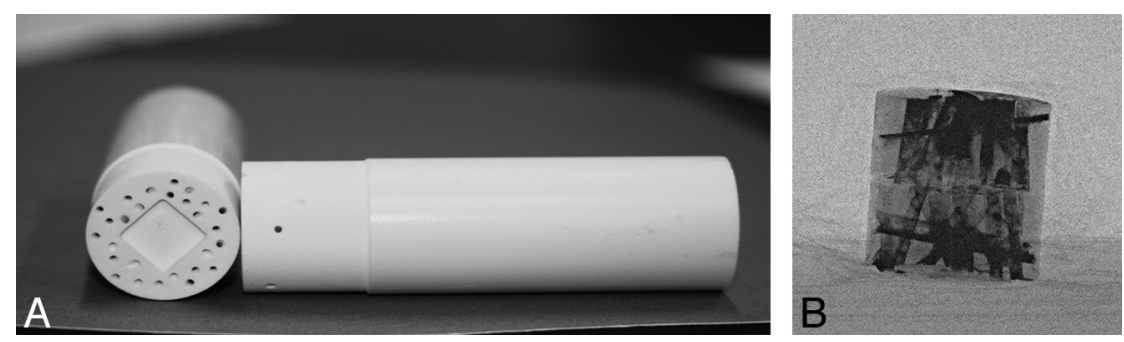

Fig 1. A, Cylindric AVM model: a plastic water phantom with a tubular perforation (model A). $B, \mathrm{X}$-ray radiography of the model $\mathrm{B}$ showing the radiopacity of the embolic agent. The film is positioned in the center of the phantom.
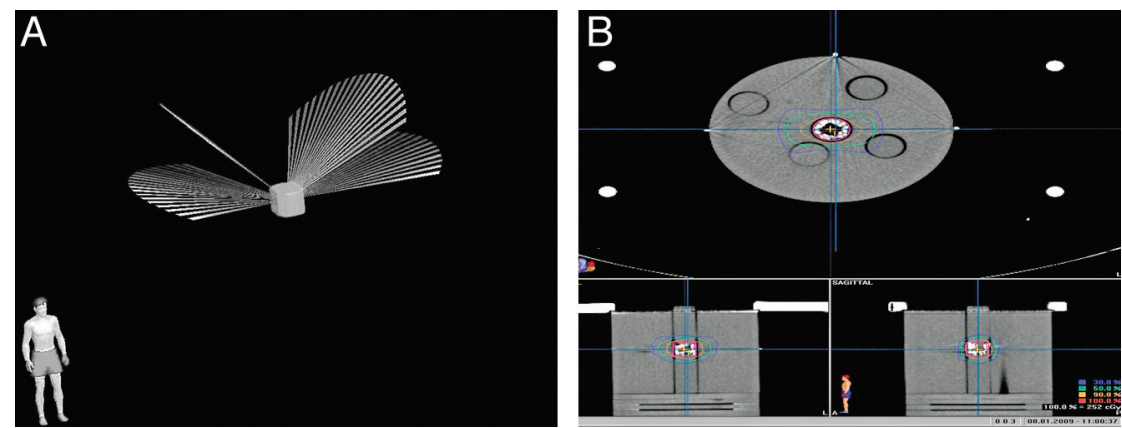

Fig 2. A, Planification of standard treatment with 4 arcs of the AVM model A. B, Isodoses curves for AVM model A positioned in a brain phantom.

vidual experiments). Following embolization with Onyx 18, this model received a mean radiation dose of $246 \pm 5$ cGy (245 and $247 \mathrm{cGy}$ ). The limitations of measurement certainty of the film ( \pm 5 cGy) showed no definitive differences in the pre- and postembolization doses delivered to the model embolized with Onyx 18.

The model $\mathrm{B}$ control received a mean radiation dose of $242 \pm 5$ cGy (245 and 238 cGy) and $254.0 \pm 5$ cGy (256 and 252 cGy) after treatment of the model with multiple varied embolization agents in common clinical use. An unexpected slight increase in radiation dose following embolization was found for model B.

\section{Discussion}

Some nonrandomized cohort publications ${ }^{2-6}$ concluded that $^{2}$ BAVM embolization before radiosurgery may have decreased the expected final rate of cure. One proposed reason was that high $\mathrm{Z}$ materials present in embolization products created a shielding effect responsible for the decrease in delivered dose. There have been supportive in vitro experiments to buttress this hypothesis: Andrade-Souza et $\mathrm{al}^{8}$ showed that a glue mixture (embucrilate + Lipiodol) was responsible for the downstream radiation reduction proportional to the Lipiodol concentration, but they only used a single beam. Against this hypothesis, Nath and Yue ${ }^{9}$ tested the shielding effect of metallic encapsulation and radiographic contrast agents for catheter-based intravascular brachytherapy, by using both photonand $\beta$-emitting radionuclides, and showed that if the shielding effect was present when low-energy photon emitters were used, it became minimal for high-energy photons (range of energy used in radiation therapy). Wilczek et $\mathrm{al}^{10}$ tested the dose perturbation induced by different stents in brachytherapy and noticed that the shielding effect exists and depends on the strut thickness and mesh attenuation rather than on the atomic number of the metal. Shtraus et $\mathrm{al}^{11}$ found that the measured attenuation of Onyx placed in a bottle was 3\% higher than the attenuation of water.

Light ion beams can also be used in radiosurgery, with clinically and radiobiologically interesting results, especially for large BAVMs. ${ }^{12}$ Measuring the dose pertubation by various high $\mathrm{Z}$ metallic devices used to shield critical structures, Das et $\mathrm{al}^{13}$ showed that electron beams gave a dual effect of dose reduction and dose enhancement, depending on the thickness and distance from the interface. Thin metal devices may enhance the dose on the transmission side and hence lose their shielding effect.

In our experiment, we did not find any attenuation of the photon beam in the center of our AVM model, either with Onyx alone or other high $\mathrm{Z}$ substances. The fact that photons are delivered through multiple arc beams and rebound of radiation on high $\mathrm{Z}$ material (backscatter effect) may explain the observed compensation for the theoretic shielding effect.

Without a randomized study, which would be very difficult to realize, it is impossible to prove that embolization has a negative effect on the outcome of BAVMs treated with radiosurgery. Nevertheless, if this effect exists, it can be explained by reasons other than a direct attenuation of the photon beam by high $\mathrm{Z}$ materials:

1) Recanalization of the embolized nidus can decrease the cure rate. ${ }^{14}$ Natarajan et $\mathrm{al}^{15}$ reported a rate of $14.3 \%$ of recanalization with Onyx at the time of the 6-month follow-up examination.

2) AVMs present a high angiogenic activity, ${ }^{16}$ which can be attenuated after radiation therapy. ${ }^{17}$ Embolization may induce hypoxia, which makes the tissue less radiosensitive and increases the angiogenic activity of the BAVM,${ }^{18,19}$ rendering it an active instead of a static lesion. ${ }^{20,21}$ Moreover, it has been shown that hypoxia-inductible factor and vascular endothelial growth factor are expressed more frequently in embolized than in nonembolized BAVMs ${ }^{18}$ in surgical specimens. 
3) A good definition of the key target volume in radiosurgery is essential. ${ }^{22} \mathrm{CT}$ and some MR imaging sequences may be useless when artifacts generated by embolic agents are substantial. As a consequence, definition of the margins of the nidus is at best an approximation, and the dose delivered to the BAVM margin is imprecise. ${ }^{11}$

\section{Conclusions}

Embolization material did not reduce the radiation dose delivered by a LINAC to the center of our experimental AVM models. The theoretic shielding effect from high $\mathrm{Z}$ materials used in embolization products is most likely compensated by backscatter effects and by multiple entry points of $\mathrm{x}$-ray beams in current clinical use. If preradiosurgical embolization reduces the obliteration rate of the BAVMs, it may occur through other mechanisms.

Disclosures: Daniel Roy—Research Support (including provision of equipment or materials). Stryker/Micrus, Details: Approximately $\$ 20,000 /$ year from each company for research support; Other Financial Relationships: Micrus, Details: $\$ 40,000$ for academic support. Alain Weill-Research Support (including provision of equipment or materials): Micrus, ev3, Details: Micrus gives financial support for our academic fund (dedicated to research and salary of fellows); for this article, ev3 provided the Onyx at no charge (they had no influence on the article), Consultant. Micrus, ev3, Details: I have a proctoring contract with these 2 companies.

\section{References}

1. Mohr JP. A randomized trial of unruptured brain arteriovenous malformations (ARUBA). Acta Neurochir Suppl 2008;103:3-4

2. Schlienger M, Atlan D, Lefkopoulos D, et al. Linac radiosurgery for cerebral arteriovenous malformations: results in $\mathbf{1 6 9}$ patients. Int J Radiat Oncol Biol Phys 2000;46:1135-42

3. Pollock BE, Flickinger JC, Lunsford LD, et al. Factors associated with successful arteriovenous malformation radiosurgery. Neurosurgery 1998;42:1239-44, discussion $1244-47$

4. Miyawaki L, Dowd C, Wara W, et al. Five year results of LINAC radiosurgery for arteriovenous malformations: outcome for large AVMS. Int J Radiat Oncol Biol Phys 1999;44:1089-106

5. Back AG, Vollmer D, Zeck O, et al. Retrospective analysis of unstaged and staged Gamma Knife surgery with and without preceding embolization for the treatment of arteriovenous malformations. J Neurosurg 2008;109(suppl): 57-64

6. Andrade-Souza YM, Ramani M, Scora D, et al. Embolization before radiosurgery reduces the obliteration rate of arteriovenous malformations. Neurosurgery 2007;60:443-51, discussion 451-52
7. Schwartz M, Sixel K, Young C, et al. Prediction of obliteration of arteriovenous malformations after radiosurgery: the obliteration prediction index. Can J Neurol Sci 1997;24:106-09

8. Andrade-Souza YM, Ramani M, Beachey DJ, et al. Liquid embolisation material reduces the delivered radiation dose: a physical experiment. Acta Neurochir (Wien) 2008;150:161-64, discussion 164. Epub 2008 Jan 23

9. Nath R, Yue N. Shielding effects of metallic encapsulations and radiographic contrast agents for catheter-based intravascular brachytherapy. Cardiovasc Radiat Med 2001;2:93-103

10. Wilczek K, Petelenz B, Strzala A, et al. Dose perturbation caused by stents: experiments with a model $90 \mathrm{Sr} / 90 \mathrm{Y}$ source. Cardiovasc Intervent Radiol 2007;30:981-91

11. Shtraus N, Schifter D, Corn BW, et al. Radiosurgical treatment planning of AVM following embolization with Onyx: possible dosage error in treatment planning can be averted. J Neurooncol 2010;98:271-76

12. Andisheh B, Brahme A, Bitaraf MA, et al. Clinical and radiobiological advantages of single-dose stereotactic light-ion radiation therapy for large intracranial arteriovenous malformations: technical note. J Neurosurg 2009;111: 919-26

13. Das IJ, Cheng CW, Mitra RK, et al. Transmission and dose perturbations with high-Z materials in clinical electron beams. Med Phys 2004;31:3213-21

14. Kwon Y, Jeon SR, Kim JH, et al. Analysis of the causes of treatment failure in gamma knife radiosurgery for intracranial arteriovenous malformations. J Neurosurg 2000;93(suppl 3):104-06

15. Natarajan SK, Ghodke B, Britz GW, et al. Multimodality treatment of brain arteriovenous malformations with microsurgery after embolization with Onyx: single-center experience and technical nuances. Neurosurgery 2008;62: 1213-25, discussion 1225-26

16. Konya D, Yildirim $\mathrm{O}$, Kurtkaya $\mathrm{O}$, et al. Testing the angiogenic potential of cerebrovascular malformations by use of a rat cornea model: usefulness and novel assessment of changes over time. Neurosurgery 2005;56:1339-45, discussion $1345-36$

17. Kilic K, Konya D, Kurtkaya $\mathrm{O}$, et al. Inhibition of angiogenesis induced by cerebral arteriovenous malformations using gamma knife irradiation. J Neurosurg 2007;106:463-69

18. Sure U, Battenberg E, Dempfle A, et al. Hypoxia-inducible factor and vascular endothelial growth factor are expressed more frequently in embolized than in nonembolized cerebral arteriovenous malformations. Neurosurgery 2004;55: 663-69, discussion 669-70

19. Akakin A, Ozkan A, Akgun E, et al. Endovascular treatment increases but gamma knife radiosurgery decreases angiogenic activity of arteriovenous malformations: an in vivo experimental study using a rat cornea model. $\mathrm{Neu}$ rosurgery 2010;66:121-29, discussion 129-30

20. Achrol AS, Guzman R, Varga M, et al. Pathogenesis and radiobiology of brain arteriovenous malformations: implications for risk stratification in natural history and posttreatment course. Neurosurg Focus 2009;26:E9

21. Mullan S, Mojtahedi S, Johnson DL, et al. Embryological basis of some aspects of cerebral vascular fistulas and malformations. J Neurosurg 1996;85:1-8

22. Valle RD, Zenteno M, Jaramillo J, et al. Definition of the key target volume in radiosurgical management of arteriovenous malformations: a new dynamic concept based on angiographic circulation time. J Neurosurg 2008;109(suppl): $41-50$ 